\title{
Hyperglycaemia stimulates pyloric motility in normal subjects
}

\author{
R Fraser, $M$ Horowitz, J Dent
}

\begin{abstract}
The motor correlates of the delay in gastric emptying produced by hyperglycaemia were investigated in $\mathbf{1 1}$ healthy volunteers. Fasting gastroduodenal motility was measured during euglycaemia (blood glucose concentration 3-5 mmol/l) and during hyperglycaemia induced by intravenous dextrose (blood glucose concentration 12-16 mmol/l). Antral, pyloric, and proximal duodenal pressures were recorded by a sleeve/sidehole manometric assembly positioned across the pylorus; with the aid of measurements of transmucosal potential difference. During hyperglycaemia there was stimulation of isolated pyloric pressure waves when compared with the euglycaemia period $(p<0.05)$. This was associated with inhibition of antral pressure waves $(p<0.05)$. In nine of the 11 subjects an episode of duodenal 'phase III like' activity occurred within 15 minutes of the onset of hyperglycaemia. It is proposed that the stimulation of localised pyloric contractions and inhibition of antral contractions contribute to the delayed gastric emptying induced by hyperglycaemia. Abnormal gastric motility in patients with diabetes mellitus may be the result of hyperglycaemia itself, rather than irreversible autonomic neuropathy.
\end{abstract}

In normal subjects, hyperglycaemia induced by intravenous glucose infusion has been associated with slowing of gastric emptying of nutrient liquid meals ${ }^{1-3}$ and a reduction in the rate of absorption of the sulphonylurea drug glipizide. ${ }^{4}$ Furthermore, a relation between delayed gastric emptying and poor glycaemic control in patients with diabetes mellitus has been shown. ${ }^{56}$ There is little information about the motor mechanisms by which hyperglycaemia slows gastric emptying. Barnett and Owyang reported that a modest increase in the blood glucose concentration is associated with suppression of antral pressure waves and antral phase III motor activity in healthy fasted volunteers. ${ }^{7}$ There are no data on the effects of hyperglycaemia on pyloric motility. Recent studies suggest that the pylorus plays an important role in the regulation of gastric emptying. ${ }^{8-10}$ Accordingly, we investigated whether hyperglycaemia stimulates pyloric motility in healthy people.

\section{Methods}

Studies were performed on 11 healthy volunteers (eight women and three men) aged between 18 and 30 years. No subject had a history of diabetes mellitus, previous gastric surgery, or was taking medication at the time of the study. Informed consent was obtained in each case and the study protocol was approved by the Human Ethics Committee of the Royal Adelaide Hospital.

\section{PROTOCOL}

Antropyloroduodenal manometry was performed in each subject with a sleeve/sidehole manometric assembly during an initial 30 minute control period of euglycaemia followed by 60 minutes of hyperglycaemia induced by intravenous infusion of $20 \%$ dextrose. No adverse effects were reported by any subject during either infusion.

Before the start of each study a cannula was inserted into an antecubital vein. Each study began in phase I or phase IIA ${ }^{11}$ of the interdigestive motor complex. Once the manometric assembly was correctly positioned, $150 \mathrm{ml}$ normal saline was given intravenously over five minutes: thereafter normal saline was infused at a rate of $150 \mathrm{ml} /$ hour. These volumes were chosen to control for the volume of intravenous dextrose required to maintin hyperglycaemia. After 30 minutes an intravenous bolus of $150 \mathrm{ml}$ of $20 \%$ dextrose was given over five minutes, followed by an infusion of $20 \%$ dextrose. Blood was drawn from a cannula placed in an antecubital vein of the arm not used for glucose infusion, and venous blood glucose concentrations were measured with BM-Test-Glycemia 20-800 strips (Boehringer-Mannheim) and a portable blood glucose meter (Refloflux IIM, Boehringer-Mannheim) at least every 10 minutes. The rate of dextrose infusion was adjusted to maintain a blood glucose concentration of approximately $14 \mathrm{mmol} / 1$ for 60 minutes. The accuracy of the blood glucose measurements was subsequently confirmed with a hexokinase technique.

The transnasally introduced manometric catheter was similar to that used in recent studies ${ }^{89}$ and incorporated a $5 \mathrm{~cm}$ long sleeve sensor that was placed astride the pylorus to monitor pyloric pressures (Fig 1). Sideholes at $1 \mathrm{~cm}$ intervals along the sleeve allowed better definition of contractions around the pylorus. Manometric sideholes at each end of the sleeve also monitored transmucosal potential difference (TMPD) in order to verify assembly position. ${ }^{8}$ Pressures and the TMPD values were recorded continuously throughout the experiment on a 12 channel chart recorder (model 7D; Grass Instrument Co, Quincy, MA, USA).

Sideholes were also located in the distal antrum 2 and $4 \mathrm{~cm}$ proximal to the sleeve and in the proximal duodenum 3 and $6 \mathrm{~cm}$ distal to the sleeve. 
Figure 1: Diagram of manometric assembly indicating the location of the sleeve sensor, transmucosal potential difference (TMPD), and manometric sidehole recording points in the antrum and duodenum.

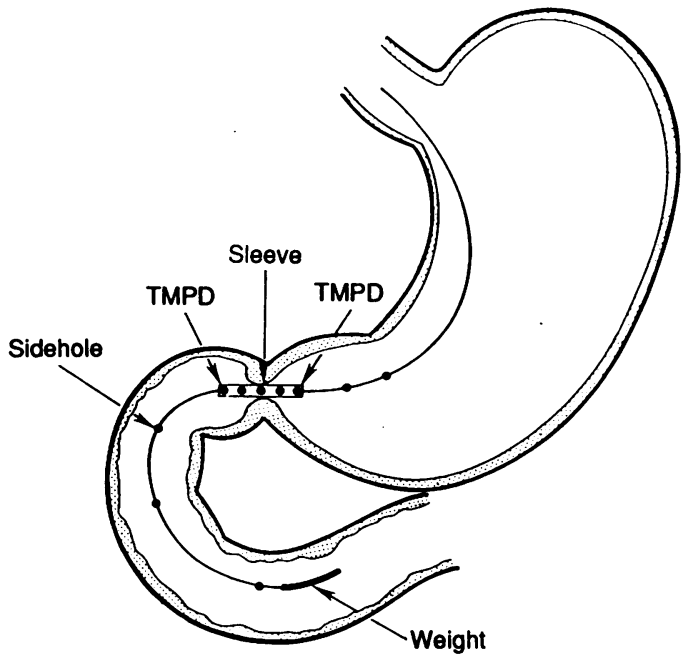

was correctly positioned across the pylorus according to previously published criteria - that is if the antral transmucosal potential difference was more negative than $-20 \mathrm{mv}$, the duodenal value was more positive than $-15 \mathrm{mv}$, and the difference between the antral and duodenal measurements was at least $15 \mathrm{mV} .^{89}$ On these criteria, the assembly was positioned correctly for more than $95 \%$ of the total recording time during the experimental period.

As in previous studies ${ }^{89}$ pressure waves were scored if their amplitude was greater than or equal to $10 \mathrm{mmHg}$. Waves recorded by the sleeve sensor were classified as isolated pyloric pressure waves when they occurred in the absence of a wave of any amplitude in the antral or duodenal transmucosal potential difference sideholes. Basal pyloric pressure was measured for each minute of the study by determining the difference between the basal pressure recorded by the sleeve and that in the most distal antral sidehole. A basal pyloric pressure of $>2 \mathrm{mmHg}$ was considered to indicate pyloric tone. ${ }^{89}$

In addition, duodenal contractions were assessed qualitatively to determine the phase of the interdigestive motor cycle. ${ }^{11}$

\section{STATISTICAL ANALYSIS}

Values were compared for each 30 minute interval (30 minutes during euglycaemia and 60

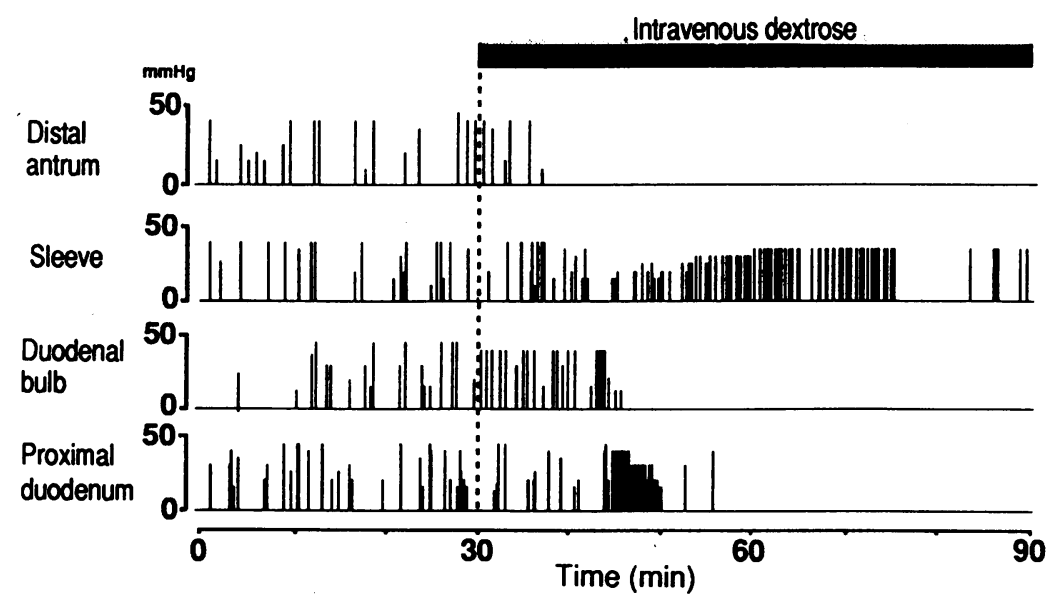

Figure 3: Time-amplitude plot of gastric antral, pyloric, duodenal bulb, and proximal duodenal pressure waves for one subject before, during, and after induction of hyperglycaemia. Amplitudes of $50 \mathrm{~mm} \mathrm{Hg}$ are at maximal pen excursions.

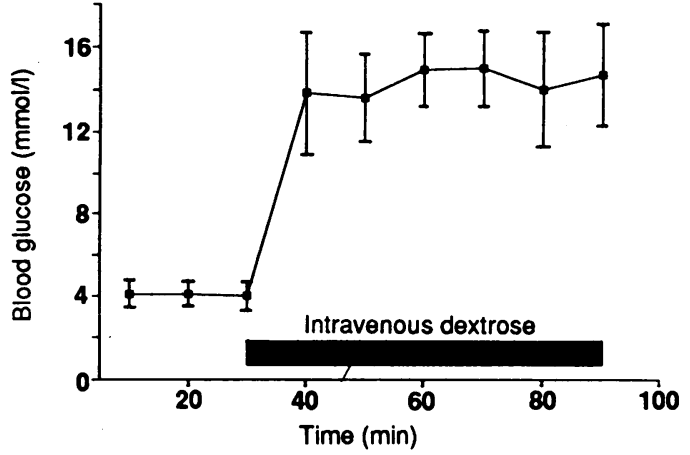

Figure 2: Blood glucose concentrations for the group during manometric measurements (values mean (SEM)).

minutes during hyperglycaemia) of the study using the two tailed Wilcoxon matched signed rank test. A p value of $<0.05$ was considered significant in all analyses. All manometric measurements are expressed as median values and interquartile range. Blood glucose concentrations are expressed as mean (SEM) values.

\section{Results}

Dextrose infusion started a median (range) of 44 (3-77) minutes after completion of the preceding duodenal phase III contractions. The blood glucose concentrations are shown in Figure 2. There was a prompt increase in the mean blood glucose concentration from $4 \mathrm{mmol} / \mathrm{l}$ to $13 \mathrm{mmol} / \mathrm{l}$ after the initial bolus of dextrose. Blood glucose concentrations were subsequently maintained at approximately $14 \mathrm{mmol} / \mathrm{l}$.

An example of a tracing from one subject showing the changes in motility patterns associated with hyperglycaemia is shown schematically in Figure 3.

The number of isolated pyloric pressure waves was significantly greater during hyperglycaemia than euglycaemia (Fig 4). There was no significant difference between the number of waves during the first 30 minutes of hyperglycaemia and the second 30 minutes of hyperglycaemia. Neither during hyperglycaemia nor euglycaemia was there evidence of pyloric tone in any subject.

There was a prompt reduction in the number of antral waves after the onset of hyperglycaemia. This reduction was sustained, almost no antral activity being observed during the second 30 minute period of hyperglycaemia (Fig 5). No antral phase III activity was seen after the induction of hyperglycaemia, but in nine of the 11 subjects an episode of high amplitude duodenal pressure waves with a rate of $10-14$ per minute and a duration of three to five minutes was observed within mean (range) 15 (4-15) minutes of the dextrose bolus (Fig 6). This motor pattern was considered as 'phase III like' as it was indistinguishable from the duodenal phase III complex of the interdigestive motor cycle. The median (range) time of appearance of this activity was 55 (11-92) minutes from the previous phase III episode of duodenal activity. Before the infusion of dextrose duodenal phase III activity was preceded by phase III activity in the gastric antrum in all 11 subjects. The duodenal phase III like activity was followed by duodenal quiescence (phase I) for between 20 and 40 minutes 
Figure 4: Number of isolated pyloric pressure waves during

hyperglycaemia and euglycaemia shown as median values and interquartile ranges for 30 minute segments of recording. There is a significant and sustained increase in the number of IPPWs during hyperglycaemia.

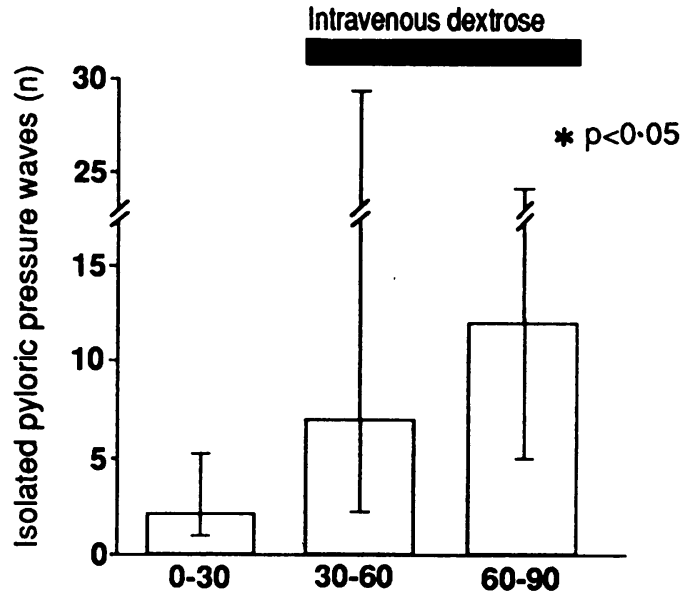

when sporadic duodenal contractions (phase II) were again recorded.

\section{Discussion}

This study adds considerably to knowledge about the effects of hyperglycaemia on upper gastrointestinal motility. Firstly, we have shown that hyperglycaemia stimulates localised pyloric contractions. Secondly, we have confirmed the potent suppressive effect of hyperglycaemia on antral motility. Lastly, we have found that duodenal phase III like activity can be stimulated by hyperglycaemia.

The intermittent obstruction to gastric outflow by pyloric contractions and the suppression of antral motility are likely to contribute to the delay in gastric emptying caused by hyperglycaemia in healthy volunteers $\mathrm{s}^{1-3}$ and in patients with diabetes mellitus. ${ }^{56} \mathrm{We}$ did not assess the effect of hyperglycaemia on the motility of the proximal stomach, which is likely to be important in gastric emptying ${ }^{12}{ }^{13}$ and may be abnormal in diabetic patients with gastroparesis. ${ }^{14}$ Further studies are also appropriate to examine the effect of lower blood glucose concentrations (within the range observed postprandially in normal subjects) on pyloric and duodenal motility. Barnett and Ouyang observed suppression of antral

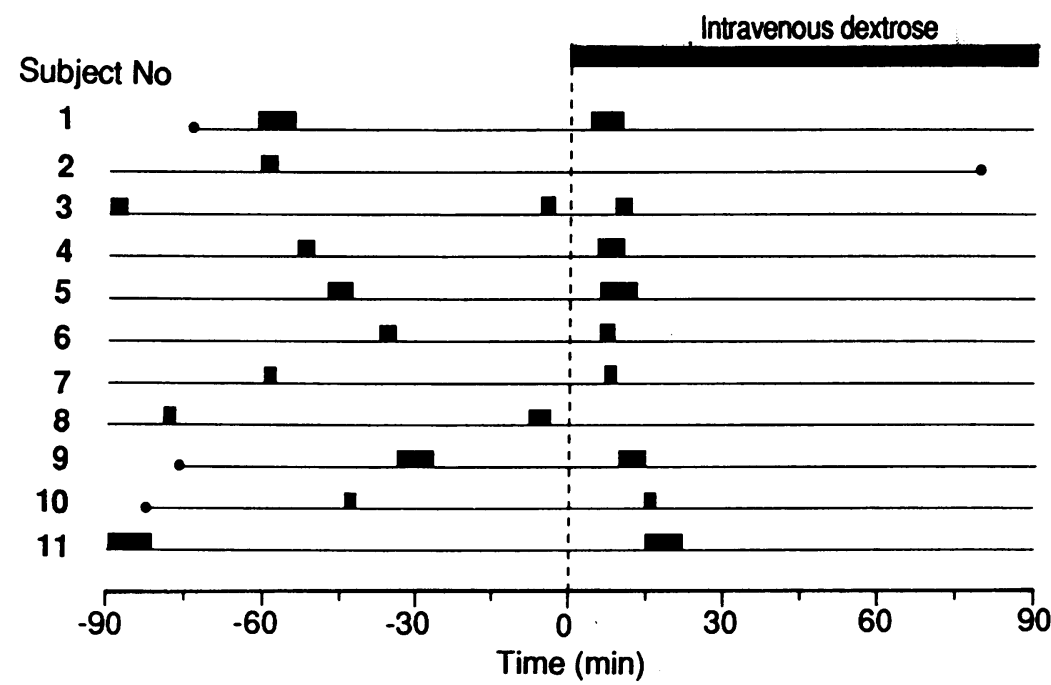

Figure 6: Timing of duodenal phase III like activity during each study. In 9 of the 11 subjects an episode of activity was seen within 15 minutes of the induction of hyperglycaemia. Length of solid bars represents length of contraction burst.

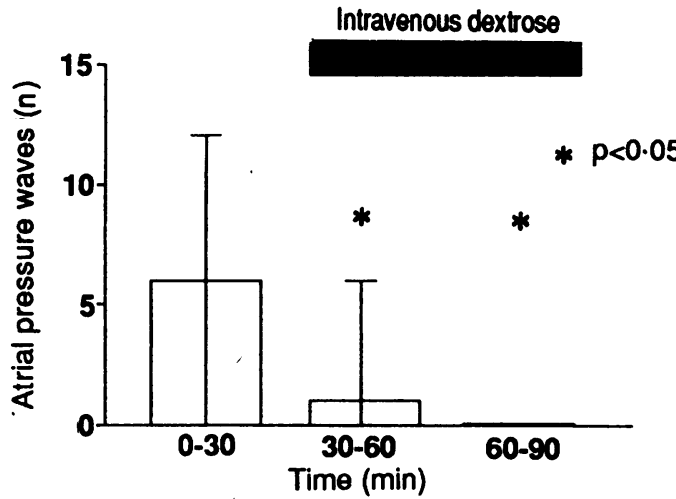

Figure 5: Number of antral pressure waves during euglycaemia and hyperglycaemia shown as median values and interquartile ranges for 30 minute segments of recording. There is a significant and sustained decrease in antral pressure waves during hyperglycaemia.

motility at a blood glucose concentration of $7 \cdot 8$ $\mathrm{mmol} / \mathrm{l}$, suggesting that changes in motor function may occur at concentrations that are seen after meals. ${ }^{7}$ Similar changes in antral and pyloric motility have been observed during intraduodenal dextrose infusion. ${ }^{15}$ Intraduodenal dextrose also results in an increase in basal pyloric pressure,${ }^{15}$ which was not observed in the present study. This may reflect a difference in stimulus potency or possibly a difference in mechanisms responsible for the mediation of pyloric tone. ${ }^{1523}$

Both neural and hormonal mechanisms may be responsible for the antral inhibition and pyloric stimulation associated with hyperglycaemia. Raised blood glucose concentrations may directly stimulate small intestinal receptors and hence lead to similar motor patterns to those seen during intraduodenal dextrose infusion. The central nervous system may modulate antropyloric motor activity via altered vagal tone, and studies in animals suggest the pylorus is under tonic neural inhibition. ${ }^{16}$ As hyperglycaemia suppresses efferent vagal activity, ${ }^{17}$ increased pyloric activity may result from interference to vagal outflow. Ascending intramural pathways have been similarly shown to modulate pyloric activity and raised blood glucose concentrations to impair nerve conduction. ${ }^{18} 19$ Intravenous glucose administration is also associated with changes in plasma concentrations of various hormones such as insulin, glucagon, and somatostatin which may influence antral and pyloric motility. ${ }^{20-22}$

The unusual duodenal phase III like activity noted during hyperglycaemia has not been reported previously. While the interdigestive motor cycle is subject to considerable inter- and intraindividual variation, the close relation between the onset of these duodenal contractions and the establishment of hyperglycaemia, and the apparent absence of a relation with the time of the previous phase III, suggests that the triggering of this activity is related to hyperglycaemia. Previous workers ${ }^{7}$ did not detect any change in the mean cycle length of the duodenal phase III activity, but did not comment on the timing of these contractions. Duodenal phase III like activity has previously been shown in response to a variety of stimuli such as intraduodenal infusion of dextrose and lipid ${ }^{1423}$ and intravenous infusion of $\beta$ endorphin, ${ }^{24}$ and during stress induced by cold, pain, ${ }^{25}$ and labyrinthine 
stimulation. ${ }^{27}$ In healthy volunteers plasma insulin concentrations rise promptly in response to an intravenous glucose load. Furthermore, intravenous administration of insulin stimulates jejunal phase III activity before any significant change in blood glucose concentration. ${ }^{28}$ Hyperinsulinaemia may therefore be important in the mediation of the duodenal phase III like activity observed in the study.

Although the blood glucose concentrations achieved in our study are not seen in normal people, they are frequently observed in patients with type 1 and type 2 diabetes mellitus. Hyperglycaemia delays gastric emptying significantly and may lead to poor glycaemic control in diabetic patients by causing discrepancies between the onset of insulin action and the release of nutrients into the intestine. ${ }^{56}$ These effects may be partly explained by our observations on antral, pyloric, and duodenal motility. It is of interest that a recent study reported an increased frequency of phasic pyloric contractions in diabetic patients with gastroparesis. ${ }^{29}$ The results of our study suggest that this and other manometric abnormalities reported in diabetic gastroparesis - for example reduced antral motility and absent antral phase III activity with normal duodenal phase III activity ${ }^{14} 3031$ - may also be partly due to hyperglycaemia itself rather than irreversible autonomic neuropathy.

This work has been published in abstract form in the Australian and New Zealand fournal of Medicine and Gastroenterology. D Fraser was supported by a Royal Adelaide Hospital Dawes Postgraduate Research Scholarship. The study was supported by grants form the National Health and Medical Research Council of Australia and the Rebecca L Cooper Medical Research Foundation Pty Ltd. The authors thank Ms S Graham for expert technical assistance.

1 Aylett P. Gastric emptying and changes of blood glucose affected by glucagon and insulin. Clin Science 1962; 22: $171-8$

2 MacGregor I, Gueller R, Watts H, Meyer J. The effects of acute hyperglycaemia on gastric emptying in man. Gastroenterology 1976; 70: 190-6.

3 Morgan LM, Tredger JA, Hampton SM, French AP, Peake JC, Marks V. The effect of dietary modification and hyperglycaemia on gastric emptying and gastric inhibitory hyperglycaemia on gastric emptying and gastric inhibitor

4 Groop LC, Defronzo RA, Luzi L, Melander A. Hyperglycaemia and absorption of sulphonylurea drugs. Lance 1989; ii: $129-30$.

5 Horowitz M, Harding PE, Maddox A, et al. Gastric and oesophageal emptying in insulin dependent diabetes mellitus. F Gastroenterol Hepatol 1986; 1: 87-113.

6 Horowitz M, Harding PE, Maddox A, et al. Gastric and oesophageal emptying in patients with type 2 (non-insulin dependent) diabetes mellitus. Diabetologia 1989; 32: 151-9.

7 Barnett J, Owyang C. Serum glucose concentration as a marke of interdigestive motility. Gastroenterology 1988; 94: 739-44.

8 Hound motor activity of the ant He gastric emptying of a solid-liquid mixed. Gastroenterology gastric emptying
1988; $94: 1285-91$
9 Heddle R, Dent J, Toouli J, Read N. Topography and measurement of pyloric pressure waves and tone in humans. Am 7 Physiol 1988; 255: (Gastrointest Liver Physiol 18) G490-7.

10 Tougas G, Anvari M, Richards D, Dent J, Somers S, Stevenson GM. Relationship of pyloric motility to transpyloric flow in healthy subjects. Gastroenterology 1987; 92: pyloric flow in
1673 [Abstract].

11 Dent J, Dodds WJ, Sekiguchi T, Hogan W, Arndorfer $R$. Interdigestive phasic contractions of the human lower oesophageal sphincter. Gastroenterology 1983; 84: 453-60.

12 Azpiroz F, Malagelada JR. Vagally mediated gastric relaxation induced by intestinal nutrients in the dog. Am $\mathcal{F}$ Physiol 1986; 251: (Gastrointest Liver Physiol 14): G727-35

13 Azpiroz F, Malagelada JR. Gastric tone measured by an electronic barostat in health and postsurgical gastroparesis. Gastroenterology 1987; 92: 934-43.

14 Malagelada JR, Rees WDW, Mazzotta LJ, Go VLW. Gastric motor abnormalities in diabetic and postvagotomy gastroparesis: effect of metoclopramide and bethanecol. Gastroparesis: effect of metoclopr

15 Heddle R, Fone D, Dent J, Horowitz M. Stimulation of pyloric motility by intraduodenal dextrose in normal subjects. Gut 1988; 29: 1349-57.

16 Allescher HD, Daniel EE, Dent J, Fox JET, Kostolanska F. Extrinsic and intrinsic neural control of pyloric sphincter in the dog. F Physiol 1988; 401: 17-38.

17 Hirano T, Niijima A. Effects of 2-deoxy-D-glucose and insulin on efferent activity in gastric nerve. Experientia $1980 ; 36$ : 1197-8.

18 Young RJ, Ewing DJ, Clarke BF. Nerve function and metabolic control in teenage diabetics. Diabetes $1983 ; 32$ $142-7$.

19 Ward JD, Barnes CG, Fisher DJ, Jessop JD, Baker RW. Improvement in nerve conduction in newly diagnosed Improvement in nerve conduction

20 Hasler W, Bowling B, Owyang C. Intraduodenal lipids induce pyloric contractions; role of cholecystokinin, the cholinergic and opiate pathways. Gastroenterology 1989; 96: A200 [Abstract].

21 Fraser $R$, Fone D, Horowitz $M$, Dent J. Effects of cholecystokinin octapeptide on antral, pyloric and duodenal motility. Australian New Zealand $\mathcal{F}$ Med 1989; 19 (suppl): 593 [A bstract].

22 Phaosawasdi K, Goppold R, Fisher R. Pyloric sphincter pressure response to insulin-induced hypoglycaemia in man Am F Physiol 1981; 241: (Gastrointest Liver Physiol 4) G321-7.

23 Heddle R, Dent J, Read NW, et al. Antropyloroduodenal motor responses to intraduodenal lipid infusion in healthy volunteers. Am f Physiol 1988; 254: (Gastrointest Liver Physiol 17) G671-9.

24 Camilleri M, Malagelada JR, Stanghellini V, Zinsmeister AR $\mathrm{Kao} \mathrm{Pai}, \mathrm{Li} \mathrm{CH}$. Dose related effects of synthetic human $\beta$-endorphin and naloxone on fed gastrointestinal motility. Am F Physiol 1986; 251: (Gastrointest Liver Physiol 14) G147-54.

25 Stangellini V, Malagelada JR, Zinsmeister AR, Go VLW, Kao PC. Stress-induced gastroduodenal motor disturbances in humans: possible humoral mechanisms. Gastroenterology 1983; 85: 83-91.

26 Thompson DG, Richelson E, Malagelada JR. Perturbation of gastric emptying and duodenal motility through the central gastric emptying and duodenal motility through the
nervous system. Gastroenterology 1982; 83: 1200-6.

27 Fone D, Horowitz M, Maddox A, Akkermans L, Read NW, Dent J. Gastroduodenal motility during the delayed gastric emptying induced by cold stress. Gastroenterology 1990; 98 $1155-61$.

28 Prasad K, Sarna S. The central and peripheral effects of insulin on migrating myoelectric complexes. Gastroenterology 1988 94: 1589 [Abstract]

29 Mearin F, Camilleri M, Malagelada JR. Pyloric dysfunction in diabetics with recurrent nausea and vomiting. Gastroenterology 1986; 90: 1919-25.

30 Camilleri M, Malagelada JR. Abnormal intestinal motility in diabetics with the gastroparesis syndrome. Eur $\mathcal{F}$ Clin Invest 1984; 13: 420-7.

31 Fox S, Behar J. Pathogenesis of diabetic gastroparesis: pharmacologic study. Gastroenterology 1981; 78: 757-63. 